\title{
On the Utilization of Spectrum Opportunity in Cognitive Radio Networks
}

\author{
Mustafa Ozger, Student Member, IEEE, and Ozgur B. Akan, Senior Member, IEEE
}

\begin{abstract}
Cognitive radio (CR) is proposed to use the spectrum opportunistically. Spectrum opportunity (SOP) can be defined as the possibility of a spectrum-aware communication between a CR transmitter and a CR receiver. Successful spectrumaware communication between the communicating CRs, which is utilization of SOP (USOP), depends on the SOP detection and the correct transmission of a packet. Spectrum sensing performance, physical channel, and network parameters affect the probability of USOP. In this letter, we characterize the probability of the USOP under different network topologies. The network topology is due to the relation between transmission ranges of licensed users and CRs. We numerically study this probability for different network parameters and topologies. We find that the characteristics of USOP highly depend on the network topology, CR sensing performance and licensed users' activities.
\end{abstract}

Index Terms-cognitive radio, spectrum opportunity, network topology.

\section{INTRODUCTION}

$\mathbf{I}$ NEFFICIENT utilization of licensed channels and overcrowded unlicensed spectrum bands lead to opportunistic use of licensed bands by unlicensed users [1]. The enabling technology of the opportunistic spectrum use is cognitive radio (CR). Nodes with CR capability can form ad hoc networks [2], named as cognitive radio networks (CRNs). Spectrum sensing and decision are the cognitive cycle functions.

Primary users (PUs) and secondary users (SUs) share the licensed spectrum band. While PUs have the privilege to utilize the spectrum band at any time, SUs use it opportunistically. We represent the SUs as CRs throughout the paper since SUs are cognitive radio nodes. CRs have spectrum opportunity (SOP) if there is no communication activity by PUs. The establishment of a communication link depends on not only the physical distance of the CRs but also the SOP occurrence [3]. Hence, the spectrum opportunity is viewed as a finite delay connectivity due to temporal dynamics of the channel in CRNs without considering the spectrum sensing performance of CRs in [3]. The authors in [4] consider only the spatial properties of successful communication in CRN. However, SOP experiences both spatial and temporal variation. Furthermore, the key point is the sensing of the spectrum vacancy correctly. Spectrum sensing performance can be enhanced by exploiting the spatial diversity of sensors and temporal diversity between the slots in a cooperative sense. The spectrum sensing performance can

The authors are with the Next-generation and Wireless Communications Laboratory (NWCL), Department of Electrical\&Electronics Engineering, Koc University, Istanbul, 34450, Turkey. e-mail: \{mozger, akan\} @ku.edu.tr.

This work was supported by The Scientific and Technological Research Council of Turkey (TUBITAK) under grant \#110E249. be increased by an improved energy detector and CRs with multiple antenna [6]. CRs with multiple antenna are costly for resource constrained networks. The authors in [7] investigate the detection of spatial-temporal opportunity. However, they do not consider successful utilization of SOP during a packet transmission. To our knowledge, there is no study in literature that jointly characterizes the SOP detection by a CR with a single radio having imperfections in the channel sensing and SOP utilization during a packet transmission.

In this letter, we investigate the probability of successful joint idle channel detection and packet transmission, which is defined as the utilization of SOP (USOP). The contribution of our paper is the determination the probability of USOP in a dynamic radio environment under imperfections in the channel sensing for different network topologies. The topologies can be categorized according to the relative transmission distance of PUs and CRs. As USOP probability defines the characteristics of the spectrum-aware communication, it can be used for developing solutions for medium access control, resource management, power control and routing in CRN.

\section{NETWORK TOPOLOGY}

Network topology affects the interaction between CRs and PUs, which also has impact on USOP. Two network topologies can be realized in CRNs [4], which we denote as $T 1$ and $T 2$.

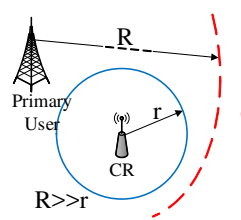

(a)

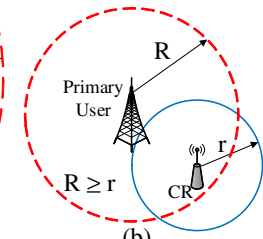

(b)
Fig. 1: Network topologies: (a) $T 1$, (b) $T 2$.

The relationship between the transmission ranges of PUs and CRs is the cause of topology difference in the network. Fig. 1 shows two different network topologies. $T 1$ is the topology, in which the transmission range of $\mathrm{PU}$ is much greater than that of CRs, hence, no spatial variation of SOP is present. There is only time variation due to the activities of the PU. T2 is the topology, in which the transmission range of $\mathrm{CRs}$ is comparable with the transmission range of PUs. Hence, there is a spatial variation due to random deployment of PUs in the network in $T 2$. Temporal activities of the PUs cause variations in time domain. Hence, $T 2$ has spatial-temporal SOP variations. 
We characterize the probability of USOP between two CRs for $T 1$ and $T 2$. The spatial and temporal variation effects are considered to fully capture the dynamics of the USOP.

\section{UtilizATION OF SPECTRUM OPPORTUNITY}

The first step of SOP utilization is the correct sensing of the spectrum. Hence, the SOP utilization depends on the performance of the spectrum sensing and network topology. We analyze the SOP under the imperfect channel sensing and the different network topologies. The second step of the utilization is to send the packet to the receiver without error.

\section{A. Imperfect Spectrum Sensing}

Energy detection is the most common method for the spectrum sensing due to its simplicity and easy implementation. However, it comprises imperfections due to channel shadowing and fading [8]. There are two types of errors, which are false alarm (FA) error and mis-detection (MD) error. FA error is due to sensing an idle channel as an occupied one. MD error is due to assuming the occupied channel as an idle one.

The SOP detection probability is the probability of sensing the channel as idle given that the channel is actually idle. We first explain the temporal activity of channel to characterize SOP. To this end, we define random variables defining the idle and busy times of PUs which are denoted as $T_{I}$ and $T_{B}$, respectively. $T_{I}$ and $T_{B}$ follow exponential distributions whose means are $\alpha$ and $\beta$, respectively [9], [10], [11]. Hence, the probability distribution functions are defined for $T_{I}$ and $T_{B}$ as $f_{T_{I}}(t)=\frac{1}{\alpha} e^{-\frac{t}{\alpha}}$, and $f_{T_{B}}(t)=\frac{1}{\beta} e^{-\frac{t}{\beta}}$, respectively. The probability that PU is idle (PU is OFF) is $P_{O F F}=\frac{\alpha}{\alpha+\beta}$. The probability that $\mathrm{PU}$ is busy ( $\mathrm{PU}$ is $\mathrm{ON})$ is $P_{O N}=\frac{\beta}{\alpha+\beta}$.

The determination of the probability of FA, $P_{F A}$, is vital to find the probability of SOP detection. If we define the event that the non-existence and existence of the PU signal as $\mathcal{H}_{0}$ and $\mathcal{H}_{1}$, respectively, $P_{F A}$ is defined as [10], $P_{F A}=\operatorname{Pr}\left[B u s y \mid \mathcal{H}_{0}\right]=P_{O F F} \mathcal{Q}\left(\frac{\delta-2 \tau_{S} B \sigma_{n}^{2}}{\sqrt{4 \tau_{S} B \sigma_{n}^{2}}}\right)$, where $B$ is the bandwidth of the channel, $\delta$ is the signal detection threshold, $\sigma_{n}$ is the noise variance, and $\tau_{S}$ is the sensing time, and $\mathcal{Q}$ is Q-function [12]. The detailed information about $P_{F A}$ and $\delta$ selection is found in [10]. In this letter, we directly consider $P_{F A}$, however, we provide the equation of $P_{F A}$ for the coherence of the paper. Vacant spectrum detection probability correctly, $P_{V}=\operatorname{Pr}\left[\operatorname{Idle} \mid \mathcal{H}_{0}\right]$, is defined as [10]

$$
P_{V}=P_{O F F}-P_{F A}=P_{O F F} \mathcal{Q}\left(\frac{2 \tau_{S} B \sigma_{n}^{2}-\delta}{\sqrt{4 \tau_{S} B \sigma_{n}^{2}}}\right) .
$$

\section{B. USOP characterization in Network Topology T1}

The temporal variation of PU activity is the only cause of the SOP change in $T 1$. There is no spatial variation since PU covers the CR in the network as seen in Fig. 1(a). The existence of SOP and its utilization between a CR transmitter and a $\mathrm{CR}$ receiver depend on the activity of the $\mathrm{PU}$, the sensing performance of the transmitter and the successful reception at the receiver. Furthermore, the packet size, $N_{b}$, and the data rate of the link, $R_{b}$, are important for USOP since there must be no PU activity during the use of the licensed spectrum band. Hence, the PU must not be active during the packet transmission interval, and it can be defined as

$$
P_{N P}=\operatorname{Pr}\left[T_{I}>\frac{N_{b}}{R_{b}}\right]=\int_{N_{b} / R_{b}}^{\infty} \frac{1}{\alpha} e^{-\frac{t}{\alpha}} d t=e^{-\frac{N_{b}}{R_{b} \alpha}} .
$$

The successful communication at the CR transmitter is realized when the transmitter detect the vacancy of the spectrum band correctly, and there is not any PU activity during the transmission activity. Hence, the USOP probability at the transmitter side can be described as

$$
\begin{aligned}
P_{t x} & =\operatorname{Pr}[\text { No PU during transmission } \mid \text { SOP detection }] \\
& =P_{N P}\left(P_{O F F}-P_{F A}\right)=P_{N P} P_{V} .
\end{aligned}
$$

(3) describes the USOP at the transmitter, and (4) shows that the USOP at the transmitter is the intersection of the event that there is no PU activity during the transmission, and the event that the transmitter successfully detects the SOP.

The probability of USOP at the receiver, $P_{r x}$, is the same as the probability of USOP at the transmitter side. The receiver must not be exposed to $\mathrm{PU}$ activities during the reception and it must detect SOP correctly. Furthermore, the communication link is unreliable due to the properties of the physical channel. Hence, the successful probability is given as [13] $P_{S}(\gamma)=(1-\Gamma(\gamma))^{N_{b}}$, where $\gamma$ is the signal-to-noise ratio (SNR) between the transmitter and the receiver which depends on the power control, $\Gamma$ is the bit error rate (BER). The expression of BER in Rayleigh fading channel for $\gamma \geq 5$ can be written as [12], [13]

$$
\Gamma_{f}(\gamma) \approx \frac{\sigma_{m}}{2 \beta_{m} \gamma}
$$

where $\sigma_{m}$ and $\beta_{m}$ depend on the modulation type. $\sigma_{m}=1$ and $\beta_{m}=2$ for binary phase shift keying modulation [12], which is used in our network. Hence, we can characterize the USOP probability as the USOP probability at the transmitter side and the receiver side without packet error. The probability of USOP can be described as

$$
P_{U S O P}^{T 1}=P_{t x} P_{r x} P_{S}=\left(\left(P_{O F F}-P_{F A}\right) P_{N P}\right)^{2} P_{S} .
$$

\section{USOP characterization in Network Topology T2}

The spatial-temporal variations in the spectrum availability affect the characterization of USOP in $T 2$. The transmission ranges of PUs and the CRs in $T 2$ are comparable as seen in Fig. 1(b), hence, there is a more dynamic radio environment. Let $R$ be the transmission range of a PU, and $r$ be the transmission range of the CRs. Fig. 2 shows a communication link between a transmitter and a receiver. The distance between the communicating nodes, $d$, is smaller than $r . A_{1}$ and $A_{2}$ are the regions that no PU activities must be present for $C R_{1}$ and $C R_{2}$, respectively.

There are two cases for USOP in T2. The union of these cases includes the conditions, which do not violate the spectrum-aware communication among CRs. These cases can be outlined as follows.

Case 1: In the first case, there must be no PUs located in the union of regions $A_{1}$ and $A_{2}$. As there are not any PUs in 
the union of these regions, the CRs must correctly detect the spectrum vacancy. Let PUs in the network be randomly deployed with a uniformly distribution whose density is $\lambda_{P U}$ [4]. The probability that there is not any PU located in the region $S(d, R)=A_{1} \cup A_{2}=2 \pi R^{2}-2 R^{2} \cos ^{-1}\left(\frac{d}{2 R}\right)+\frac{d}{2} \sqrt{4 R^{2}-d^{2}}$ can be defined as

$$
P_{1}=\operatorname{Pr}\left[\text { No PUs in } A_{1} \cup A_{2}\right]=e^{-\lambda_{P U} S} .
$$

The probability that $C R_{1}$ successfully detects the spectrum vacancy can be written as $P_{O F F}-P_{F A}$. $C R_{2}$ must sense the vacant channel correctly, and this probability is the same as the probability of correct spectrum vacancy detection for $C R_{1}$. Furthermore, the packet must be received without error, and this probability is $P_{S}$. Hence, the probability of Case 1 is written as

$$
P_{C 1}^{T 2}=P_{1}\left(P_{O F F}-P_{F A}\right)^{2} P_{S} .
$$

Case 2: In the second case, there may be PUs located in the region $S(d, R)$, and the number of PUs located in the region $S(d, R)$ is denoted by $N_{P U, S}$. The PUs located in region $S(d, R)$ affect the communication since the communication activity of a PU in this region interferes with the transmitter or the receiver or both. Hence, all the PUs located in this region must be in OFF state during the transmission of packet. The probability that there are $k$ PUs in region $S$ can be given as $\operatorname{Pr}\left[N_{P U, S}=k\right]=e^{-\lambda_{P U} S} \frac{\left(\lambda_{P U} S\right)^{k}}{k !}$. PUs are distributed uniformly in the network, and the density of PUs is denoted by $\lambda_{P U}$. Hence, the probability that there are $k$ PUs in the region $S$ is given according to a Poisson distribution. We can assume that the network size is infinity, and the number of PUs in the network can be approximated as infinity.

Let $Q_{P U, S}$ be the state of the PUs in the region $S$. If $Q_{P U, S}=0$, the PU nodes in the region $S$ are in OFF state during the packet transmission. Hence, we can denote the USOP probability in Case 2 as

$$
\begin{aligned}
P_{2} & =\operatorname{Pr}\left[N_{P U, S}>0 \cap Q_{P U, S}=0\right] \\
& =\operatorname{Pr}\left[N_{P U, S}>0\right] \operatorname{Pr}\left[Q_{P U, S}=0 \mid N_{P U, S}>0\right] \\
& =\sum_{k=1}^{\infty} \frac{e^{-\lambda_{P U} S}\left(\lambda_{P U} S\right)^{k}}{k !} P_{N P}^{k} \\
& =e^{-\lambda_{P U} S}\left(\sum_{k=0}^{\infty} \frac{\left(\lambda_{P U} S P_{N P}\right)^{k}}{k !}-1\right) \\
& =e^{-\lambda_{P U} S\left(1-P_{N P}\right)}-e^{-\lambda_{P U} S} .
\end{aligned}
$$

The probability of no active PUs for Case 2 in $T 2$ is written in a conditional form as in (10). As in (11), the uniform distribution of PUs in the network causes a Poisson distribution for possible PUs in the region $S$, where $k$ represents the number of PUs in the region $S$, and its range is from one to infinity since we are considering the case that there are one or more PUs in the region $S$. Furthermore, the number of PUs in the network may increase to infinity as the network size is assumed to be infinity. We use the Taylor expansion of exponential number in (12) and (13). For a successful SOP detection and its utilization, the PUs in the region $S$ must be OFF during the communication. The transmission must also be carried without error in Rayleigh fading channel, whose

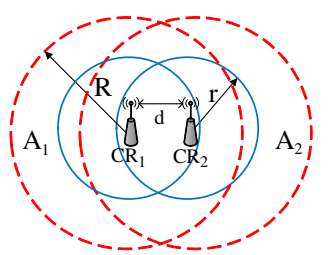

Fig. 2: A communication link between $C R_{1}$ and $C R_{2}$.

probability is $P_{S}$. The probability of USOP in $T 2$ for Case 2 can be written as

$$
P_{C 2}^{T 2}=P_{2}\left(P_{O F F}-P_{F A}\right)^{2} P_{S}
$$

since both the transmitter and the receiver should sense SOP and utilize the idle channel successfully. The overall USOP probability for $T 2$ is found by the summation of the probabilities of cases $C 1$ and $C 2$ as follows,

$$
P_{U S O P}^{T 2}=P_{C 1}^{T 2}+P_{C 2}^{T 2}=P_{S}\left(P_{1}+P_{2}\right)\left(P_{O F F}-P_{F A}\right)^{2} .
$$

\section{NumERICAL RESUlTS}

In this section, we study the probability of USOP under different conditions and network topologies. As explained in Section III, $P_{U S O P}$ depends on the network system parameters according to the network topology. Unless otherwise stated, we set the system parameters as $R_{b}=100 \mathrm{Kbps}, N_{b}=500 \mathrm{bits}$, $P_{O F F}=0.9, P_{F A}=0.1, \alpha=100 \mathrm{msec}, d=10 \mathrm{~m}, R=$ $20 m, \gamma=30 \mathrm{~dB}$, and $\lambda_{P U}=0.0001 P U / m^{2}$, which are on the order the values in [9], [10], [13], [11]. In our first study, we investigate the USOP probability for different system parameters which are $N_{b}, R_{b}, \alpha, \gamma$ and $P_{O F F}$ as seen in Fig. 3 . These parameters are selected due to their ability to change the channel characteristics and the network as in [10], [11].

We first investigate the effects of $R_{b}$ and $N_{b}$. As $N_{b}$ increases, $P_{N P}$ in (2) decreases, and also $P_{S}$ decreases. Hence, $P_{U S O P}^{T 1}$ in (6) decreases. For $R_{b}=50 \mathrm{kbps}, 25 \%$ decrease occurs as the greatest change for a 750 bits change in $N_{b}$. $P_{U S O P}^{T 2}$ decreases due to the increase in $N_{b}$ in (13) for $T 2$ as seen in Fig. 3(a). This decrease is due to the increase in $N_{b}$. The approximate change is $18 \%$ for $P_{U S O P}^{T 2}$ for all $R_{b}$. Also, as $\alpha$ increases, $P_{N P}$ in (2) increases. Hence, $P_{U S O P}^{T 1}$ decreases as seen in Fig. 3(b). However, the effect of $\alpha$ is at most $5 \%$ for $T 2$. However, $\alpha$ has positive effect on $P_{U S O P}^{T 2}$ due to (13). $\alpha$ is the mean inactive time of PUs, and an increase in $\alpha$ increases the probability of no PU activity during the communication effort. OFF state of PU is actually means an opportunity for a CR. It has power control to change their SNR, $\gamma$. For SNR values smaller than $15 \mathrm{~dB}$, the channel is in deep fade and the communication is almost impossible. However, $P_{U S O P}^{T 1}$ and $P_{U S O P}^{T 2}$ reach almost 0.5 for $30 \mathrm{~dB}$ as seen Fig. 3(c). The quality of channel increases and the fading effects become negligible. An increase in $R_{b}$ decreases the communication time, hence, the probability that communicating nodes expose to PU activity decreases, which also increases $P_{N P}$. This increase causes an increase in $P_{U S O P}^{T 1}$ for $T 1$ due to (2) and a decrease in $P_{U S O P}^{T 2}$ for $T 2$ due to (13).

The network parameters such as $R, \lambda_{P U}$ and $d$ affect the SOP probability only in $T 2$. Hence, we study the effect of 


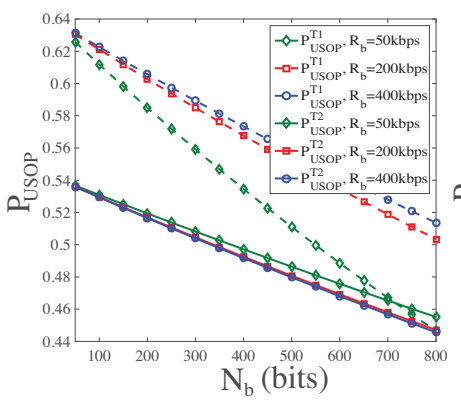

(a)

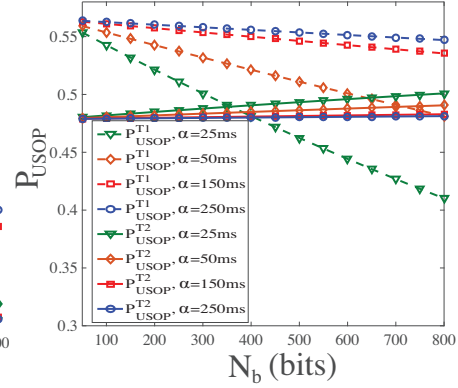

(b)

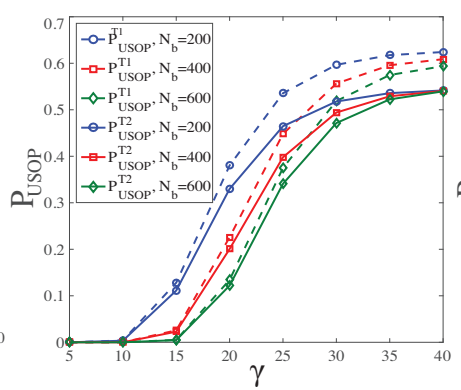

(c)

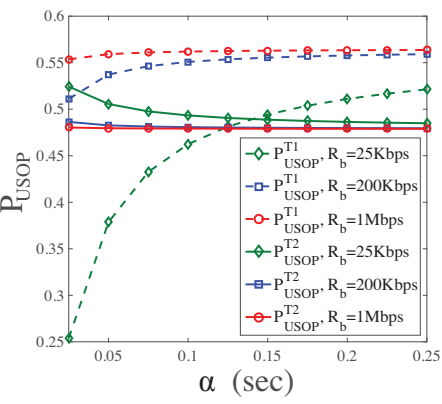

(d)

Fig. 3: Probability of USOP under different topologies vs. (a) $N_{b}$, (b) $N_{b}$, (c) $\gamma$, and (d) $\alpha$.

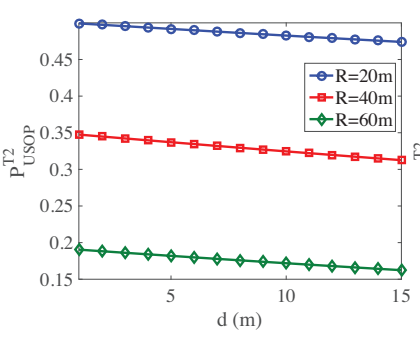

(a)

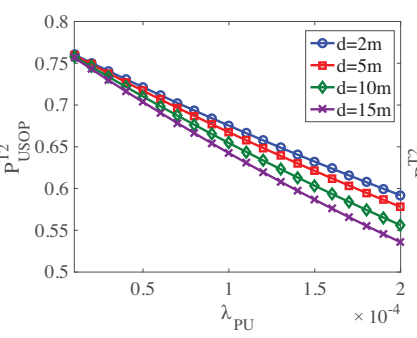

(b)

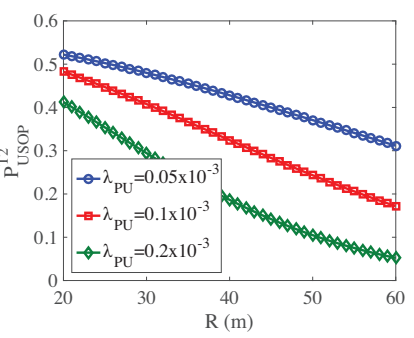

(c)

Fig. 4: Probability of USOP under network topology $T 2, P_{U S O P}^{T 2}$, vs. (a) $d$, (b) $\lambda_{P U}$, (c) $R$.

these parameters on $P_{U S O P}^{T 2}$. As we increase $R$, the CRs become more exposed to the activities of PUs. Hence, this effect decreases $P_{U S O P}^{T 2}$. The effect of $d$ is small since $R$ is much greater than $d$. The effects of $d$ and $R$ can be seen in Fig. 4(a). $\lambda_{P U}$ is a significant parameter since it affects the number of PUs located in the communication area of the CRs. As we increase $\lambda_{P U}$, there would be more PU activity in the communication area. Hence, it decreases $P_{U S O P}^{T 2}$ as seen Fig. 4(b). For instance, $P_{U S O P}^{T 2}$ decreases nearly $30 \%$ when $\lambda_{P U}$ increases from $25 \times 10^{-6}$ to $200 \times 10^{-6}$. The effect of $R$ can be seen in Fig. 4(c) in a more profound way. The increase in $R$ causes an increase in $S(d, R) . P_{U S O P}^{T 2}$ decreases as $S(d, R)$ increases in (15). Nearly $85 \%$ decrease in $P_{S O P}^{T 2}$ is seen when $\lambda_{P U}=200 \times 10^{-6}$. Hence, $R$ highly affects $P_{U S O P}^{T 2}$.

\section{Conclusion}

In this letter, we characterize the USOP probability under different network topologies. We find that this probability depends on the network topologies, the sensing performance of CRs, the channel conditions, and the PU activities. We observe the effects of them on the USOP probability in our numerical analysis. This probability can be used for proposing new solutions and protocols in medium access control, routing, transport and resource allocation schemes as it provides a probabilistic view of the spectrum-aware communication.

\section{REFERENCES}

[1] I. F. Akyildiz, W. Y. Lee, M. C. Vuran, and S. Mohanty, "NeXt generation / dynamic spectrum access / cognitive radio wireless networks: A survey," Computer Networks (Elsevier), Sept. 2006.
[2] I. F. Akyildiz, W.-Y. Lee, and K. R. Chowdhury, "CRAHNs: Cognitive radio ad hoc networks," Ad Hoc Netw., vol. 7, no. 5, pp. 810-836, Jul. 2009.

[3] W. Ren, Q. Zhao, and A. Swami, "Temporal Traffic Dynamics Improve the Connectivity of Ad Hoc Cognitive Radio Networks," IEEE/ACM Trans. on Netw., vol. 22, no. 1, Feb. 2014.

[4] M. Bradonjic and L. Lazos, "Graph-based Criteria for Spectrum-aware Clustering in Cognitive Radio Networks," Ad Hoc Networks (Elsevier), vol. 10, no. 1, pp. 75-94, Jan. 2012.

[5] G. Ding, F. Song, Q. Wu, and J. Wang, "Joint Spatial-Temporal Spectrum Sensing in the Presence of Reporting Errors," in Proc. of IEEE WCNC 2014, Istanbul, Turkey, pp. 707-712.

[6] A. Singh, M. Bhatnagar, and R. Malik, "Performance of an Improved Energy Detector in Multi-Hop Cognitive Radio Networks," IEEE Trans. on Vehicular Technology, 2015.

[7] Q. Wu, G. Ding, J. Wang, and Y.-D. Yao, "Spatial-Temporal Opportunity Detection for Spectrum-Heterogeneous Cognitive Radio Networks: TwoDimensional Sensing," IEEE Trans. on on Wireless Comm., vol. 12, no. 2, Feb. 2013.

[8] X. Zhou, G. Y. Li, D. Li, D. Wang, and A. C. K. Soong, "Probabilistic Resource Allocation for Opportunistic Spectrum Access," IEEE Trans. on Wireless Comm., vol. 9, no. 9, Sep. 2010.

[9] S. Huang, X. Liu, and Z. Ding, "Opportunistic Spectrum Access in Cognitive Radio Networks," in Proc. of IEEE INFOCOM'08, pp. 21012109.

[10] W.-Y. Lee and I. F. Akyildiz, "Optimal Spectrum Sensing Framework for Cognitive Radio Networks," IEEE Trans. on Wireless Comm., vol. 7 , no. 10 , pp. 3845-3857, Oct. 2008.

[11] M. C. Oto and O. B. Akan, "Energy-Efficient Packet Size Optimization for Cognitive Radio Sensor Networks," IEEE Trans. on Wireless Comm., vol. 10, no. 4, pp. 1544-1553, Apr. 2012.

[12] A. Goldsmith, Wireless Communications. Cambridge Univ. Press, 2005.

[13] R. Zhang and J.- M. Gorce, "Optimal Transmission Range for Minimum Energy Consumption in Wireless Sensor Networks," in Proc. IEEE WCNC 2008, pp. 757-762. 